\title{
Kinetic Monitoring of Block Copolymer Self-Assembly using In-Situ Spectroscopic Ellipsometry
}

Connor R. Bilchak, ${ }^{1,2}$ Shivajee Govind, ${ }^{1}$ Guillermo Contreas, ${ }^{1}$ Boris Rasin, ${ }^{2}$

Shawn M. Maguire, ${ }^{2}$ Russell J. Composto, ${ }^{2,{ }^{*}}$ and Zahra Fakhraai ${ }^{1,}$

${ }^{1}$ Department of Chemistry, University of Pennsylvania, Philadelphia, PA, USA.

${ }^{2}$ Department of Materials Science and Engineering, University of Pennsylvania, Philadelphia, PA, USA.

*Correspondence to: fakhraai@sas.upenn.edu, rcomposto@seas.upenn.edu 


\section{Materials and Methods}

All materials were used as received without further purification. Poly(styrene)-b-poly(methyl methacrylate) (PS-b-PMMA) and poly(styrene)-b-poly(2-vinyl pyridine) (PS-b-P2VP) were purchased from polymer source. The molecular weights of each block and their dispersity indices are shown in Table S1. The solvents chloroform and toluene were purchased from Sigma-Aldrich. Silicon wafers (100) purchased from Virginia Semiconductor were cut into $\approx 1 \mathrm{~cm} \times 1 \mathrm{~cm}$ squares. Silicon wafers were cleaned in piranha solution (70:30 vol\%, $\left.\mathrm{H}_{2} \mathrm{SO}_{4}: \mathrm{H}_{2} \mathrm{O}_{2}\right)$ at $80^{\circ} \mathrm{C}$ for 20 minutes, rinsed three times with deionized water, and dried under nitrogen gas. The polymer thin films were deposited via spin casting at 1000rpm from 4 wt.\% solutions in toluene. Film thicknesses of the dry films were measured via spectroscopic ellipsometry as well as reflectometry (Filmetrics F20) and were $300-350 \mathrm{~nm}$ for each film.

Ex-situ annealing was performed using chloroform vapor. $30 \mathrm{ml}$ of chloroform was added to the bottom of a 150ml glass jar with a two-inch Teflon pedestal to support the sample. The jar was capped, and the solvent vapor allowed to build up for 30 minutes to ensure saturation. The jar was then uncapped, the sample was placed on the pedestal, and quickly recapped. After the film was annealed for a targeted time, the jar was uncapped, and the film quickly removed from the jar. The jar was then re-sealed, and the solvent vapor pressure allowed to re-equilibrate for at least 30 minutes before any subsequent solvent annealing. The films were allowed to dry under ambient conditions for at least 16 hours before any further experiments to ensure complete solvent removal. Film thickness measurements before and after solvent annealing conducted with both SE and reflectometry, agree to within $\pm 5 \mathrm{~nm}$, indicating that the film has returned to its dry state.

\section{Grazing Incidence Small Angle X-ray Scattering (GISAXS)}

GISAXS was performed on a Xenocs Xeuss 2.0 with a sample to detector distance of $6.36 \mathrm{~m}$ with a $\mathrm{Cu}-\mathrm{k} \alpha$ source, providing an available $q$-range of $0.003-0.09 \dot{A}^{-1}$. The exposure time to collect each scattering image was 6 hours. The incident angle between the X-rays and the sample surface was fixed at $0.22^{\circ}$. Data were analyzed using Foxtrot Software. The domain spacing was obtained from the maximum scattering intensity as a function of either $q_{z}$ (for perpendicular lamellae) or $q_{x, y}$, (for parallel lamellae).

For the PS-b-PMMA orientational control experiments, the polymer was assembled using thermal annealing. The BCP thin films were heated to $190^{\circ} \mathrm{C}$ under vacuum for 48 hours and then cooled to room temperature. The orientation of the domains was controlled by adding a PS-b-PMMA surface passivating layer to the silicon substrate, which results in lamellae aligned perpendicular to the substrate, as shown previously (ref. ${ }^{50}$ in main text). The passivating layer is $\approx 5 \mathrm{~nm}$ of $25 \% / 25 \% / 50 \%$ PS (6.4k), PMMA (8.0k) and PS-b-PMMA (5k-b-5k). Parallel lamellae are formed by thermally annealing the BCP without the passivating layer at $190^{\circ} \mathrm{C}$ for 48 hours; perpendicular lamellae were formed by similar thermal annealing but with the added passivating layer. The inter-lamellar spacing of the perpendicularly-aligned samples, was measured using both surface AFM and GISAXS to be $39 \mathrm{~nm}$; the values taken from the two techniques are within experimental error. Films assembled in the absence of this layer will assemble parallel to the substrate, as shown in Figure 3 of the main text. 


\section{$\underline{\text { In-situ SVA measurements }}$}

In-situ solvent vapor annealing (SVA) measurements were conducted using a custom apparatus designed to fit under the ellipsometer for in-situ measurements (Schematics and a picture of the set up shown in Figure S1). All the equipment and parts of the SVA system, except for the Mass Flow Controllers (MFCs), were custom built using glass, Teflon, or stainless steel to avoid mixing or interaction with the organic solvents. The stainless steel swelling chamber (Figure S1A) has two openings, made with quartz glass windows. These glass windows are at an angle of $70^{\circ}$ to the normal of base of the ellipsometer, to allow the incident and reflected lights of the ellipsometer to be orthogonal to the glass window and at $70^{\circ}$ normal to the sample. Details for fitting the data while removing the background of the quartz window are explained in the 'Ellipsometry Measurements and Data Fitting Procedure' section.

Ultra-high purity nitrogen gas was used as a carrier gas in the SVA system. The flow of the nitrogen gas was controlled by Aalborg GFC17 MFCs (Orangeburg, NY). The Taps (T1, T2, T3 and T4) shown in Figure S1A were used to stop the flow of gas or vapor in a particular line while the switches (S1, S2 and S3) were used to change the direction of flow between the two lines when needed.

To generate solvent vapor, the taps along line 1 (T1 and T2) were opened to allow nitrogen gas to bubble through the solvent (chloroform in this study). The process of bubbling a gas through a solvent, also known as sparging, promotes evaporation of the solvent. The solvent vapor was given approximately 5 minutes to equilibrate in the mixing container and directed to the fume hood along lines 3 and 6, whilst skipping the swelling chamber. Switches S1, S3 and S2 were then flipped to allow the solvent to go through lines 4, 5, and 6, via the swelling chamber and into the fume hood. The thickness and properties of the thin films were monitored in-situ via ellipsometry as the solvent penetrated the film. For all in-situ measurements, a constant flow of $(300 \pm 1) \mathrm{mL} /$ minof $\mathrm{N}_{2}$ was held throughout the SVA system which generates a solvent vapor pressure of $\approx 110$ Torr for this system. When the solvent annealing process was complete, taps 1 and 2 along line 1 were closed and taps 3 and 4 along line 2 were opened to flush out the solvent from the swelling chamber and to quench the solvent annealed films inside the swelling chamber with a flow of $(300 \pm 1) \mathrm{mL} / \mathrm{min}$ dry nitrogen.

\section{Determining the Solvent Vapor Pressure}

Assuming that nitrogen does not dissolve into the solvent during the sparging process and that the nitrogen or the non-polar solvent behaves ideally at room temperature, the ratio of molar flow rate to pressure is a constant. In other words, the ratio of the molar flow rate of the solvent, $\dot{M}_{\text {sol }}$ to its vapor pressure, $P_{\text {sol }}$ is given by equation 1 where $\dot{M}_{\text {nit }}$ is molar flow rate of the nitrogen, and $P_{\text {nit }}$ is its respective pressure.

$$
\frac{M_{\text {sol }}}{P_{\text {sol }}}=\frac{M_{\text {nit }}}{P_{\text {nit }}}
$$

Since it is an open system, one can assume that the net pressure of the system is at atmospheric pressure or 760 Torr. The net pressure was also measured by a dual valve pressure gauge connected to the outlet of the nitrogen gas. Therefore, equation 1 can be rewritten in the form of equation 2 to give the value of vapor pressure as a function of molar flow rates of the solvent and nitrogen flow.

$$
\frac{M_{\text {sol }}}{P_{\text {sol }}}=\frac{M_{\text {nit }}}{760-P_{\text {sol }}}
$$

A solvent trap was incorporated into the SVA system (Figure S1A) in order to measure the solvent vapor pressure. The temperature of the solvent trap was lowered to at least $195 \mathrm{~K}$ using dry ice. This was a sufficiently low temperature since the normal boiling point of chloroform is $334 \mathrm{~K}$. In order to calculate the solvent vapor pressure, taps T1 and T2 along line 1 were opened to generate the solvent vapor, which was 
directed along lines 4 and 7, via the swelling chamber and solvent trap, by adjusting the switches S1 and $\mathrm{S} 3$ accordingly. The mass of solvent, $m$ grams collected over a period, $t$ minutes ( $t>120$ minutes) for a flow of Nitrogen of $\dot{Q} \pm 1 \mathrm{~mL} / \mathrm{min}$ bubbled through a solvent of molecular weight $M_{w}$, can be used to determine the molar flow rate of the solvent, $\dot{M}_{\text {sol }}$ using equation 3 . The molar flow rate of the Nitrogen, $\dot{M}_{\text {nit }}$ can determined by equation 4 , where $\rho$ is the density of Nitrogen gas at room temperature.

$$
\begin{aligned}
& \dot{M}_{\text {sol }}=\frac{m}{t \cdot M_{w}} \\
& \dot{M}_{\text {nit }}=\frac{\dot{Q} \rho}{M_{w}}
\end{aligned}
$$

The vapor pressure of the solvent, $P_{\text {sol }}$, at a flow rate $\dot{Q}$ of the nitrogen flow and at room temperature, can be determined using equation 2,3 , and 4 . For a nitrogen flow of $(300 \pm 1) \mathrm{mL} / \mathrm{min}$ sparged through chloroform for this particular set up, the vapor pressure of chloroform generated was $\approx 110$ Torr at room temperature.

\section{Ellipsometry Measurements and Data Fitting Procedure}

For ex-situ measurements, the optical properties of the films were measured in five distinct locations across the surface using a J.A. Woollam Spectroscopic Ellipsometer (M-2000, Lincoln, NE) and the results were averaged. Each acquisition was 1 second in length.

Ellipsometry measures the ellipsometric angles $\Psi$ and $\Delta$ as a function of wavelength $(\lambda)$, which are related to the ratio of the reflection coefficients of the film at two different polarizations by:

$$
\frac{r_{p}}{r_{s}}=\Psi e^{i \Delta}
$$

Where $r_{p}$ and $r_{s}$ are the reflection coefficients for the $\mathrm{p}$ and s polarized light, respectively. This data is fit with various optical models to represent the film, with the goal to lower the mean squared error (MSE) between the data and the model using the smallest number of adjustable parameters. The data were fit to a three-layer model; the bottom two layers represented the silicon substrate and a $1 \mathrm{~nm}$ native oxide layer. The third layer represented the polymer film and was modeled using the Cauchy dispersion relations:

$$
n=A+\frac{B}{\lambda^{2}} \quad, \quad k=0
$$

The values of $A, B$, film thickness, roughness, and the substrate angle offset (a measure of the imperfection of the sample alignment) were set as fit parameters. Both $A$ and $B$ were restricted to positive values to obtain physically realistic data. For in-situ measurements, the angle offset was fit for the $t=0$ min measurement (i.e., before solvent annealing) and fixed for the duration of the experiment. Data in the wavelength range 500-1600 $\mathrm{nm}$ were used for fitting. The data were first fit using $A, B$, film thickness, film roughness, and angle offset as fitting parameters. This typically resulted in the MSE to be less than 2 for both pure polymer films and as-cast BCPs, indicating an isotropic structure. If the MSE after this fitting procedure was $>3.5$, the data was fit to a uniaxial birefringence model (equation 7).

For example, SE spectra collected on a cylinder-forming PS-b-P2VP (48k-b-136k) is shown in Figure S2A; In the absence of a priori knowledge of the film structure, the ellipsometry data is fit assuming that the refractive index of the film is isotropic; however, applying this model shows significant deviations from the experimental data for ordered films and results in a large MSE values of $\approx 8-12$. For these films, we assume uniaxial birefringence:

$$
\delta n=n_{z}-n_{x, y}
$$


Where $n_{x, y}$ and $n_{z}$ are the in-plane and out of- plane indices of refraction. Since values of $B$ are typically much less than $A$, a wavelength-independent birefringence model is used such that $n_{z}-n_{x, y} \approx A_{z}-A_{x, y}=$ $\delta A$. The inclusion of birefringence as a fit parameter for the as-cast film does not significantly affect the MSE, indicating the polymer is isotropic directly after spin-casting. Including uniaxial birefringence in the self-assembled thin films reduces the MSE from $\approx 8-12$ to $\approx 2-3$, indicating its importance in properly modelling the optical properties of the assembled BCP. The errors between the data and the isotropic or anisotropic model fits are shown in Figures S2B and S2 C respectively. Additionally, we compared the model fits for a single incident light angle $\left(70^{\circ}\right)$ and multiple angles $\left(55^{\circ}-75^{\circ}\right.$ in multiples of 5) and found that the measured birefringence between the two were similar to within 0.001 . This indicates that for film thicknesses used in this study, using a single angle of incidence was sufficient to model the film's optical birefringence. The incident angle was fixed at $70^{\circ}$ for all in-situ experiments. In order to properly model the optical properties of the films measured in-situ, the effect of the quartz windows on the data were also considered. Each sample used for in-situ measurements was first measured in ambient conditions without the quartz windows in place. The film thickness, optical constants, and angle offset were then fit using this data. A second measurement of the same film, at the same spot, was then taken with the quartz windows in the path of the beam; any difference in the data between the first and second measurement is the result of the addition of the windows. This effect was then corrected for by fitting the data using an offset in the ellipsometric angle $\Delta$, while fixing the film thickness, optical constants, and angle offset obtained based on the first measurement. The addition of the quartz windows did not increase the fit MSE more than 3-5\% of its value without the windows. The $\Delta$ effect was then kept constant during the in-situ measurements.

For the ordered BCP films, fitting the data with a model which assumes a refractive index gradient in the direction of film thickness did not significantly reduce the MSE (Figure S3), which indicates that the optical properties of the BCP film are reasonably constant in the direction normal to the film's surface. This provides further evidence that the assembled BCP domains persist throughout the entire film.

\section{Autocorrelation Function Calculation from Surface AFM}

Image filtering and analysis was performed using Gwyddion software. The images were leveled using the mean field subtraction function and scan rows aligned using a median filter. Background noise was removed using a $4^{\text {th }}$ order polynomial background. The radial autocorrelation functions were generated using Gwyddions built-in processor.

\section{Calculating the maximum birefringence.}

The maximum birefringence of a thin film $\left(\delta n_{\max }\right)$ of a perfectly ordered copolymer features is computed by adapting the theoretical predictions for birefringence in uniaxially anisotropic crystals ${ }^{47}$ :

$$
\delta n_{\max }{ }^{2}=n_{z}^{2}-n_{x, y}^{2}=\frac{f_{1} f_{2}\left(n_{1}^{2}-n_{2}^{2}\right)^{2}}{f_{1} n_{2}^{2}+f_{2} n_{1}^{2}}
$$

Where $f$ indicates the volume fraction and $n$ the refractive index, and the subscripts 1 and 2 denote either PS or P2VP. This equation assumes a positive birefringence, i.e., the optical axis is parallel to the axis of the domains and is therefore valid for structures oriented perpendicular to the substrate. In the case of structures orientated perpendicular to the optical axis (i.e., parallel to the substrate) the sign of the equation is negative. The $A$-values of the two polymers were also obtained through $S E$ measurements of $\approx 100 \mathrm{~nm}$ thick spin-casted films of the two homopolymers. At least 10 individual films were measured/modeled and their results averaged. We note that we are assuming a wavelength-independent birefringence here where $\delta n=\delta A$. The measured values of $A$ for the homopolymers used in this study are listed in table S2. 
Table S1: Physical properties of the BCPs used in this work. DI=dispersity index. The block $\mathrm{M}_{\mathrm{n}}$ 's were used to calculate the relative volume fractions in the $\mathrm{BCP}\left(\mathrm{f}_{\mathrm{A}}=\mathrm{M}_{\mathrm{n}, \mathrm{A}} / \mathrm{M}_{\mathrm{n}, \text { total }}\right)$

\begin{tabular}{|c|c|c|c|c|c|}
\hline Polymer & $\begin{array}{c}\text { Block A M } \\
\text { (kDa) }\end{array}$ & $\begin{array}{c}\text { Block B M } \\
\text { (kDa) }\end{array}$ & DI & $\begin{array}{c}\text { Equilibrium } \\
\text { morphology }\end{array}$ & $\boldsymbol{\delta}_{\text {max }}$ \\
\hline PS-b-P2VP & 48 & 136 & 1.08 & Cylinders & 0.0016 \\
\hline PS-b-P2VP & 133 & 132 & 1.15 & Lamellae & 0.0011 \\
\hline PS-b-PMMA & 38.0 & 36.8 & 1.11 & Lamellae & 0.048 \\
\hline
\end{tabular}

Table S2: Refractive indices of the pure polymers used in this work.

\begin{tabular}{|c|c|}
\hline Polymer & A \\
\hline PS & $1.588 \pm 0.001$ \\
\hline P2VP & $1.565 \pm 0.001$ \\
\hline PMMA & $1.511 \pm 0.001$ \\
\hline
\end{tabular}


A

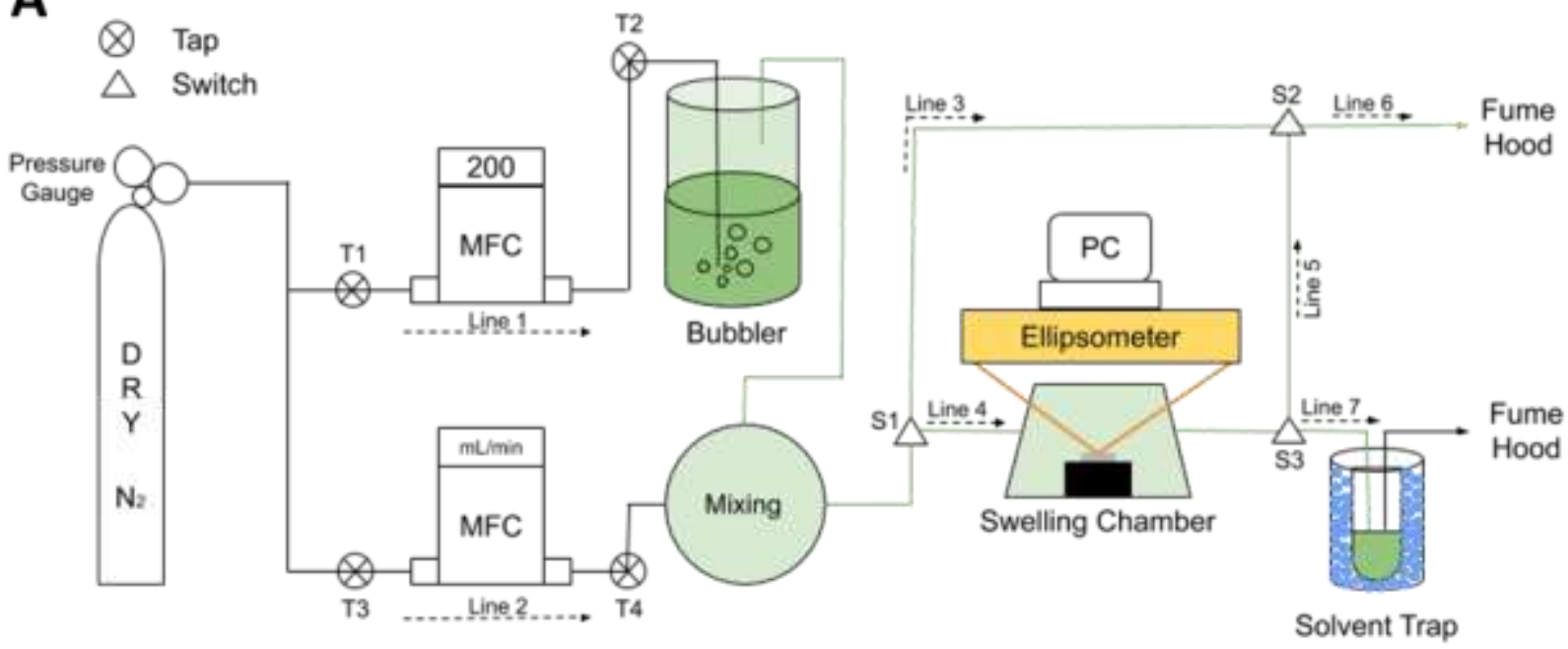

B

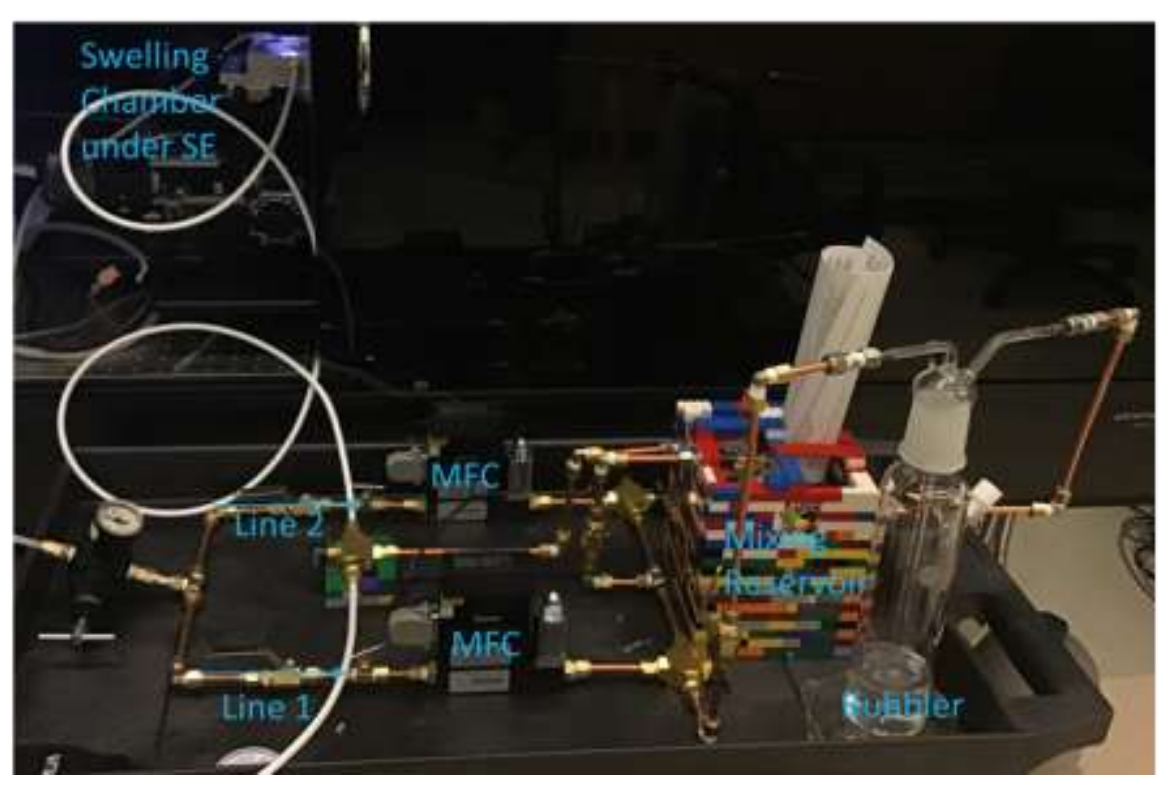

Figure S1: (A) schematic diagram and (B) experimental setup of the SVA system. 

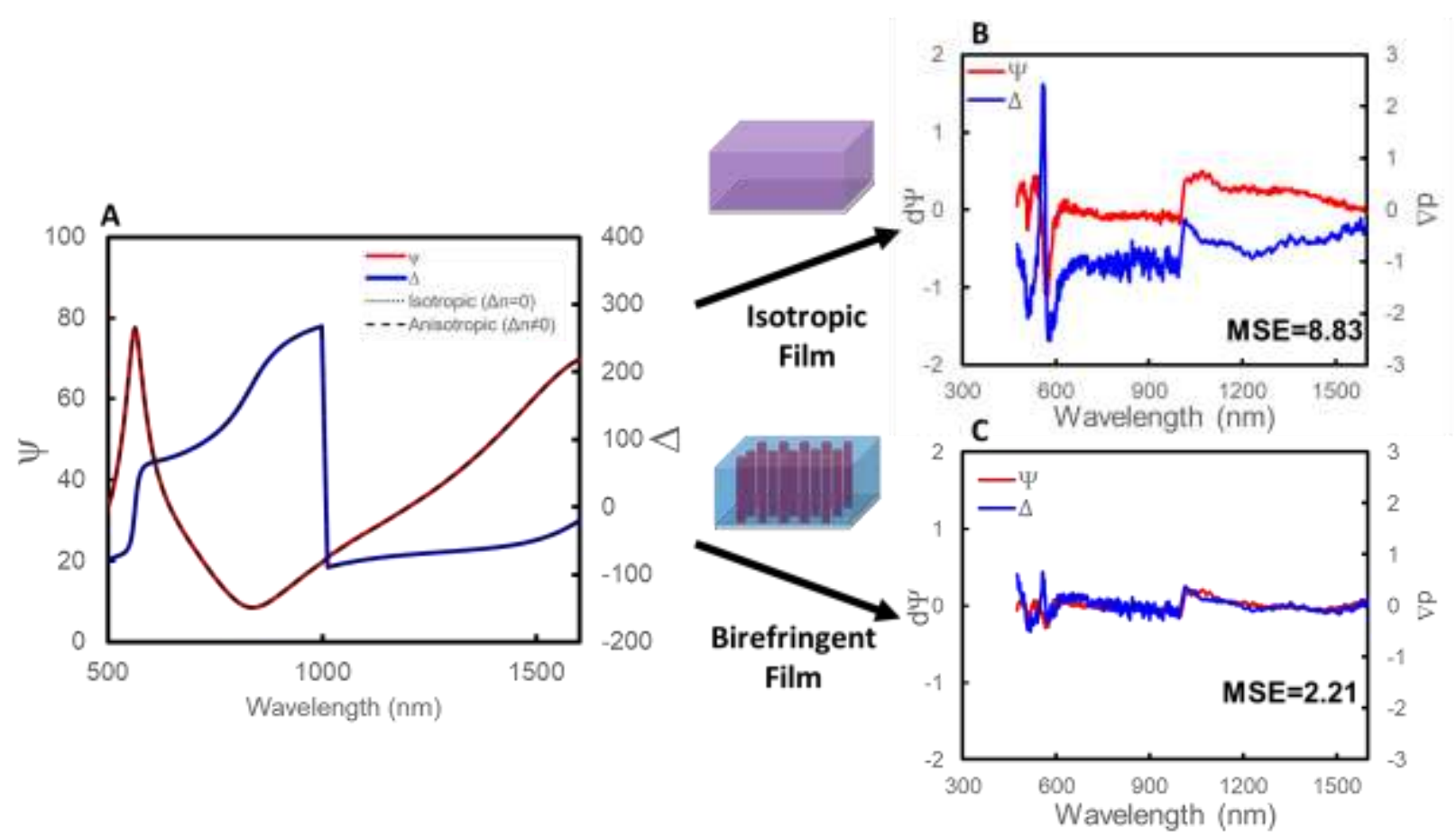

Figure S2: Optical characterization of cylinder-forming PS-b-P2VP BCP thin films using spectroscopic ellipsometry. (A) Ellipsometric angles $\Psi$ and $\Delta$ versus wavelength of a cylinder-forming BCP film after 5 minutes of chloroform annealing. The black dotted line represents a fit to the data assuming an isotropic model (i.e., the Cauchy relation) while the black dashed line represents an anisotropic model. (B,C) MSE error between the fit and measured $\Psi$ (red) and $\Delta$ (blue) using (B) an isotropic model for the film refractive index and (C) a uniaxial anisotropic model. MSE value for each fit is reported in the inset. MSE is significantly reduced upon fitting an anisotropic model (C) and the difference between the data and the model is reduced at all wavelengths. 
A

\begin{tabular}{|c|c|c|c|c|c|}
\hline Parameter & Ideal & Roughness & Grading & $\begin{array}{c}\text { Roughness \& } \\
\text { Grading }\end{array}$ & $\begin{array}{c}\text { Uniaxial } \\
\text { Birefringence }\end{array}$ \\
\hline MSE & 11.391 & 9.355 & 9.944 & 9.195 & 3.525 \\
\hline Roughness & N/A & $5.33 \pm 0.318 \mathrm{~nm}$ & $\mathrm{~N} / \mathrm{A}$ & $4.08 \pm 0.429 \mathrm{~nm}$ & $1.50 \pm 0.169 \mathrm{~nm}$ \\
\hline A & $1.563 \pm 0.00058$ & $1.563 \pm 0.00046$ & $1.567 \pm 0.00059$ & $1.565 \pm 0.00061$ & $1.566 \pm 0.00022$ \\
\hline B & $0.0093 \pm 9.89 \mathrm{E}-05$ & $0.0099 \pm 8.82 \mathrm{E}-05$ & $0.0084 \pm 0.000106$ & $0.0094 \pm 0.000145$ & $0.0133 \pm 3.91 \mathrm{E}-5$ \\
\hline dZ & N/A & N/A & N/A & N/A & $0.0122 \pm 0.0002$ \\
\hline \% Inhomogeneity & N/A & N/A & $-3.64 \pm 0.271$ & $-1.53 \pm 0.344$ & N/A \\
\hline Thickness\# 1 & $329.18 \pm 0.22 \mathrm{~nm}$ & $328.87 \pm 0.18 \mathrm{~nm}$ & $328.68 \pm 0.19 \mathrm{~nm}$ & $328.76 \pm 0.18 \mathrm{~nm}$ & $327.23 \pm 0.09 \mathrm{~nm}$ \\
\hline Angle Offset & $0.142 \pm 0.0159$ & $0.117 \pm 0.0129$ & $0.0471 \pm 0.0156$ & $0.0837 \pm 0.0148$ & $0.0668 \pm 0.0058$ \\
\hline
\end{tabular}

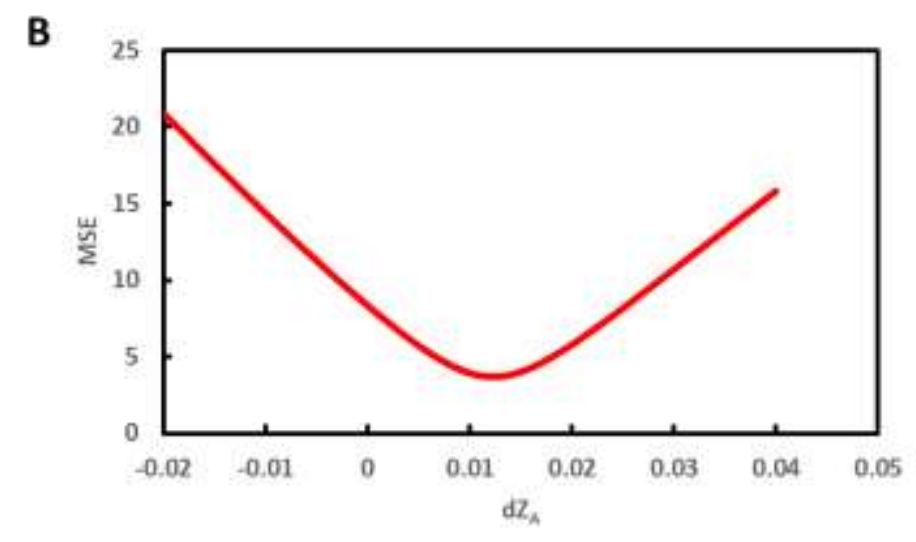

Figure S3: Results of SE fitting. (A) table comparing recorded fit parameters and MSE's for a variety of film models; ideal (fits only A,B, film thickness, and angle offset), added roughness, added grading, added roughness and grading, and uniaxial birefringence. Roughness is modeled as a thin effective medium of the polymer film and air (50/50\%); grading assumes that the refractive index of the film is allowed to vary linearly with film depth. Adding birefringence significantly reduces the MSE. (B) sensitivity of the model fit to variances in the film birefringence $\left(d Z_{A}=A_{z}-A_{x, y}\right)$. 


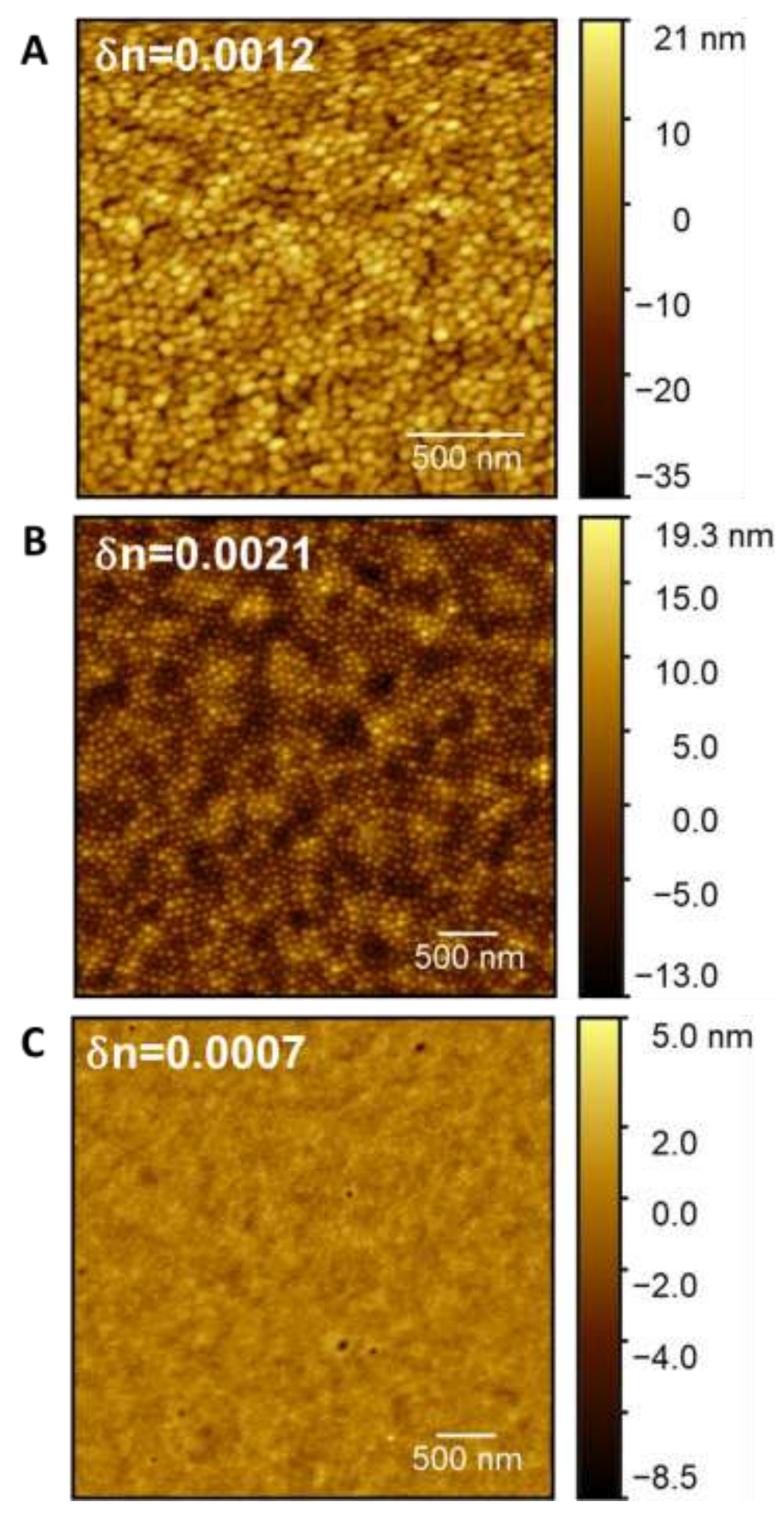

Figure S4: AFM images of As-Cast BCPs. Initial morphologies of (A) 48k-b-136k PS-b-P2VP, (B) 133kb-132k PS-b-P2VP, and (C) 38k-b-36k PS-b-PMMA. 
A

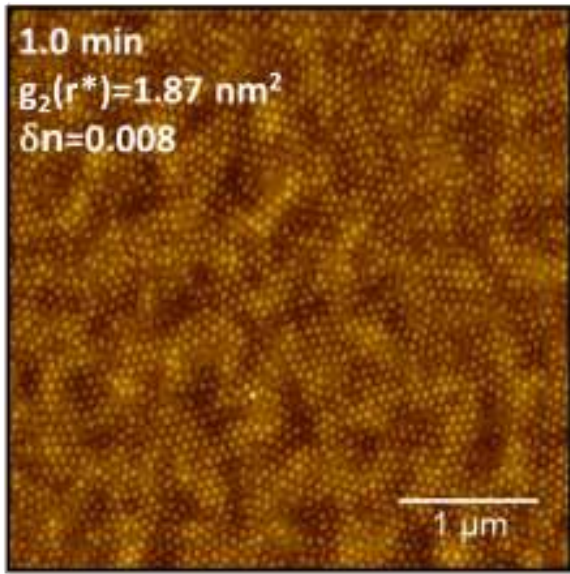

C

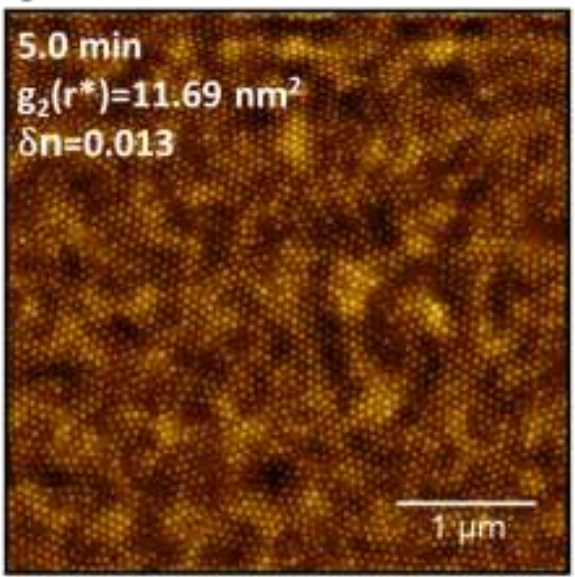

E

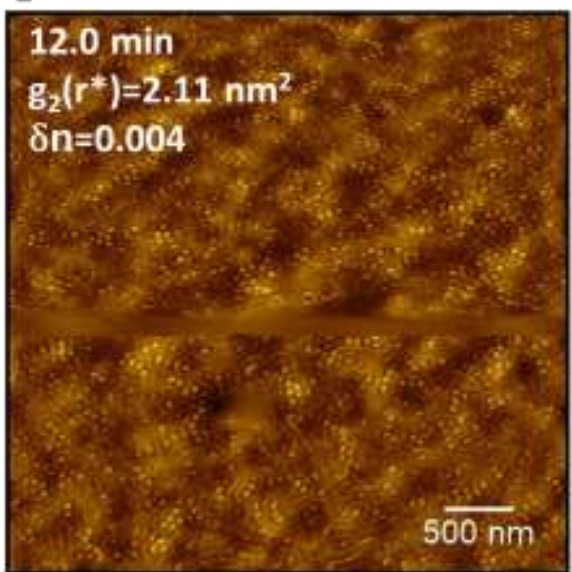

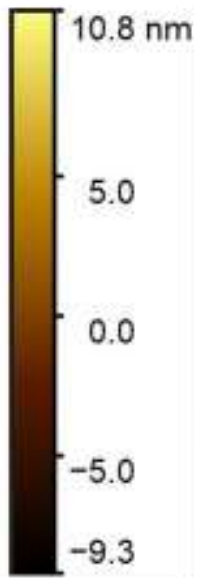

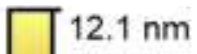

10.0

8.0

6.0

4.0

2.0

0.0

$-2.0$

$-4.0$

$-7.4$
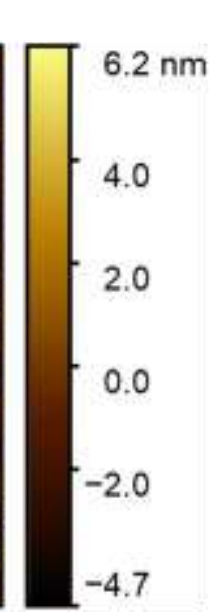

B

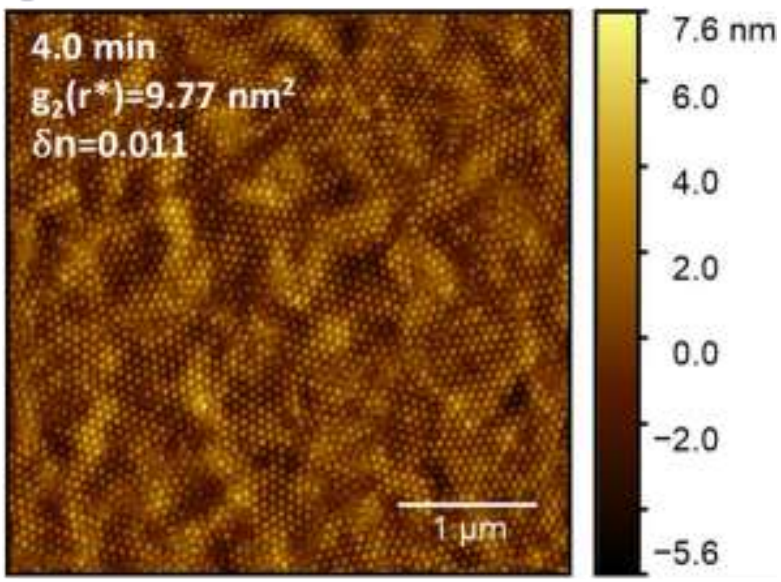

D

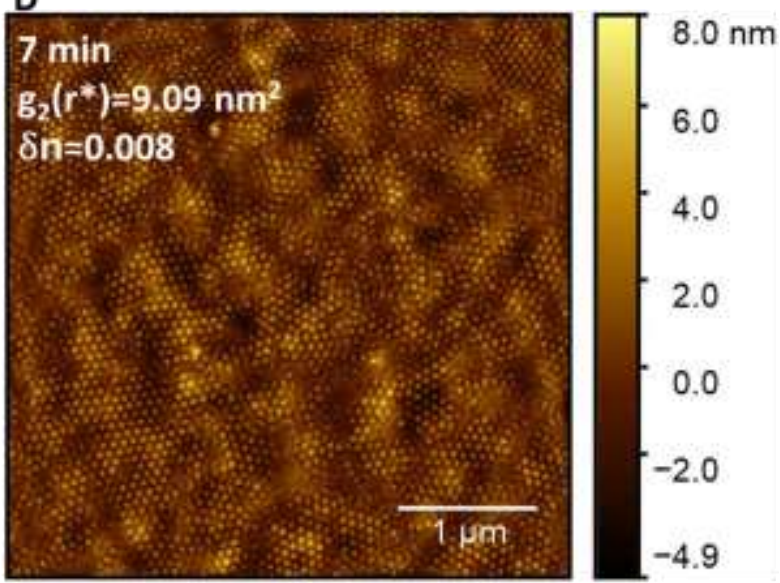

F

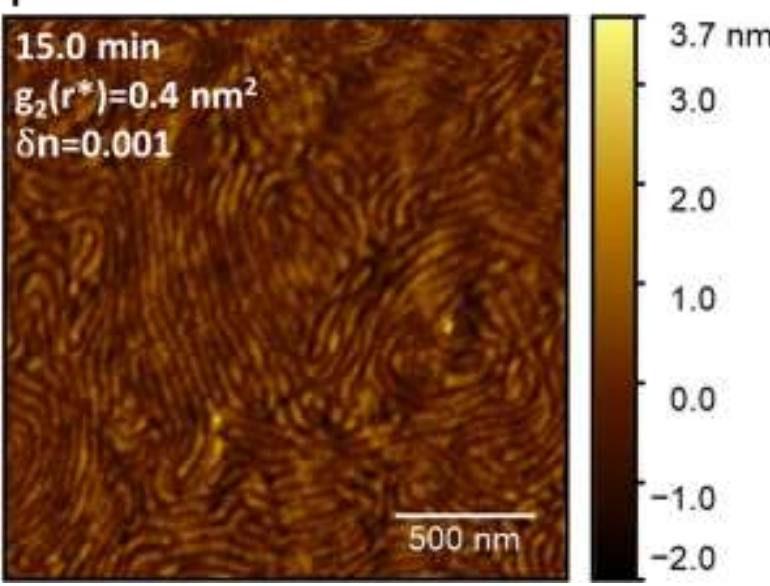

Figure S5: AFM images of 48k-b-136k PS-b-P2VP as a function of chloroform annealing time. The initial film thicknesses are all between $320 \mathrm{~nm}$ and $345 \mathrm{~nm}$. 


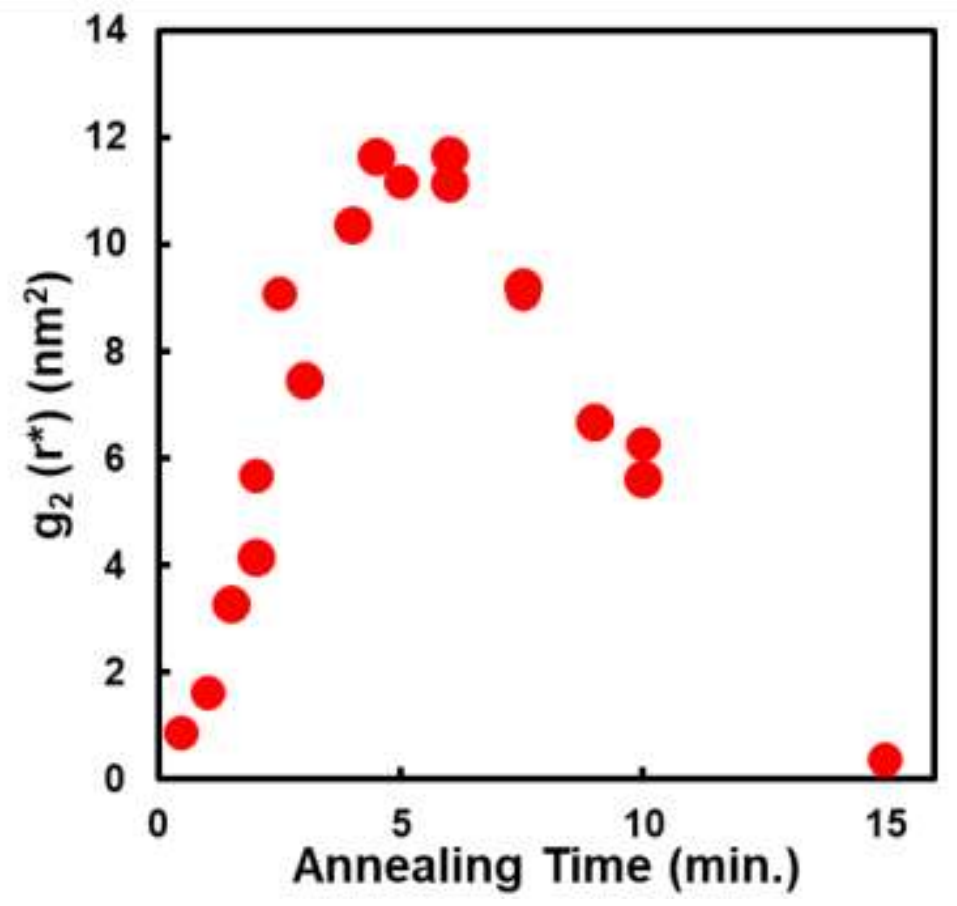

Figure S6: Change in $\mathrm{g}_{2}\left(\mathrm{r}^{*}\right)$ for the cylinder-forming PS-b-P2VP (48k-b-136k) films as a function of exsitu chloroform solvent annealing time. 

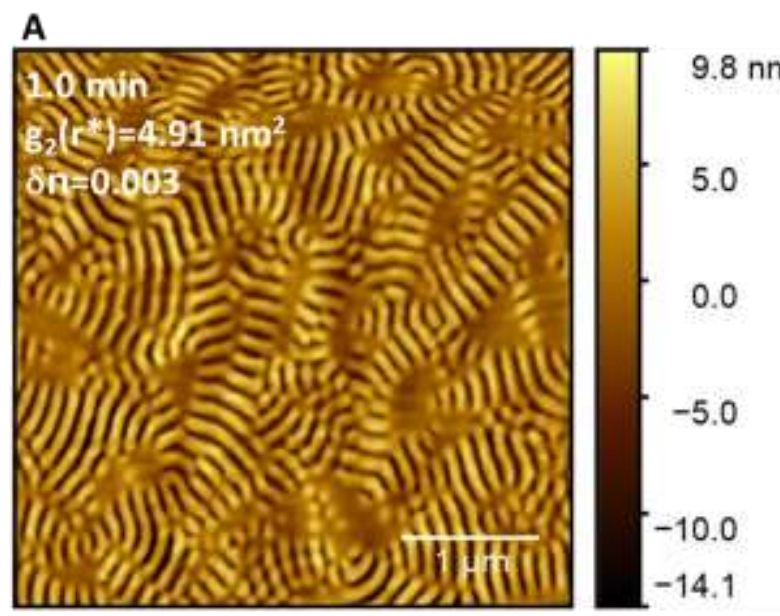

B
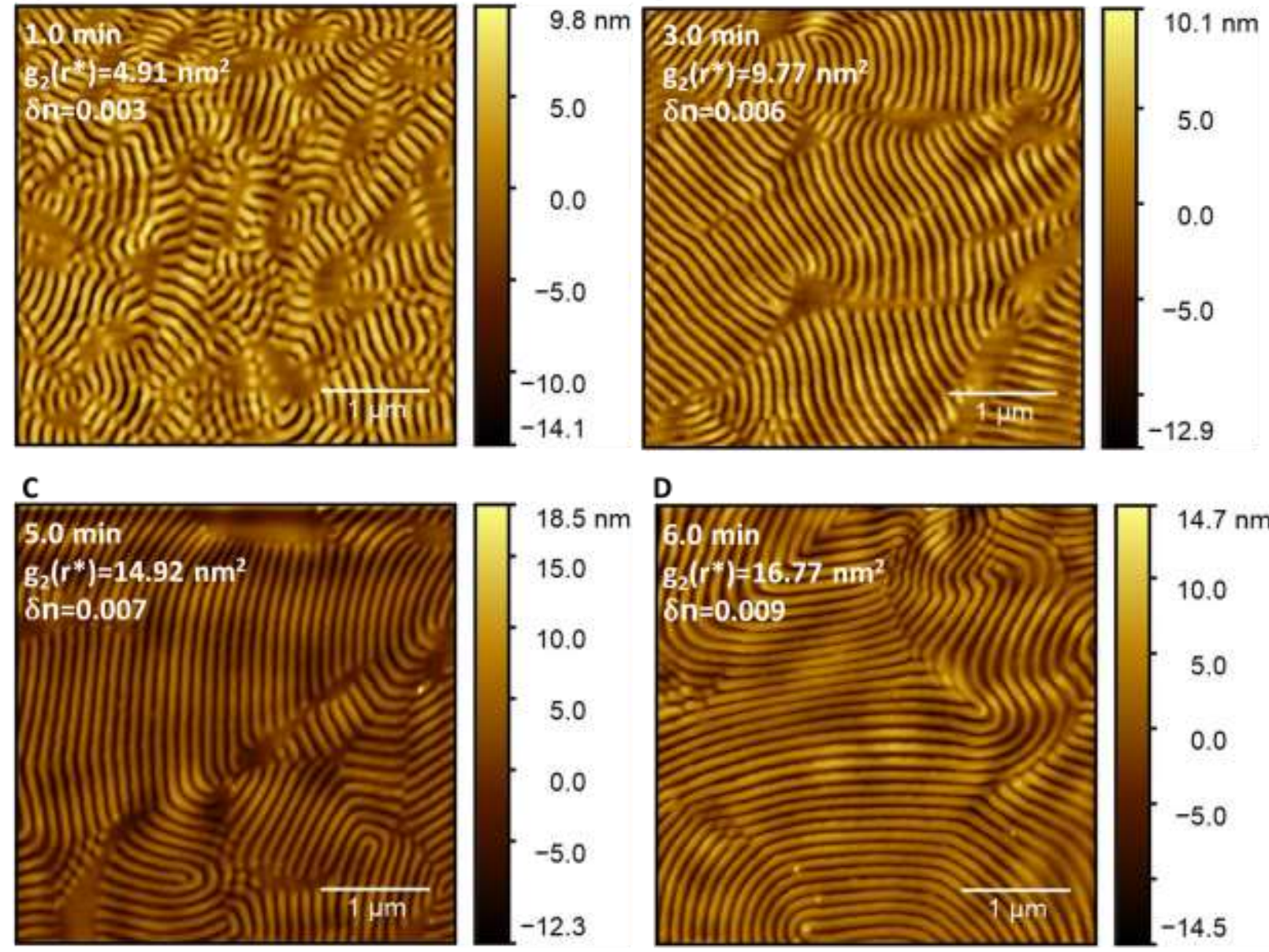

D

$\mathrm{E}$
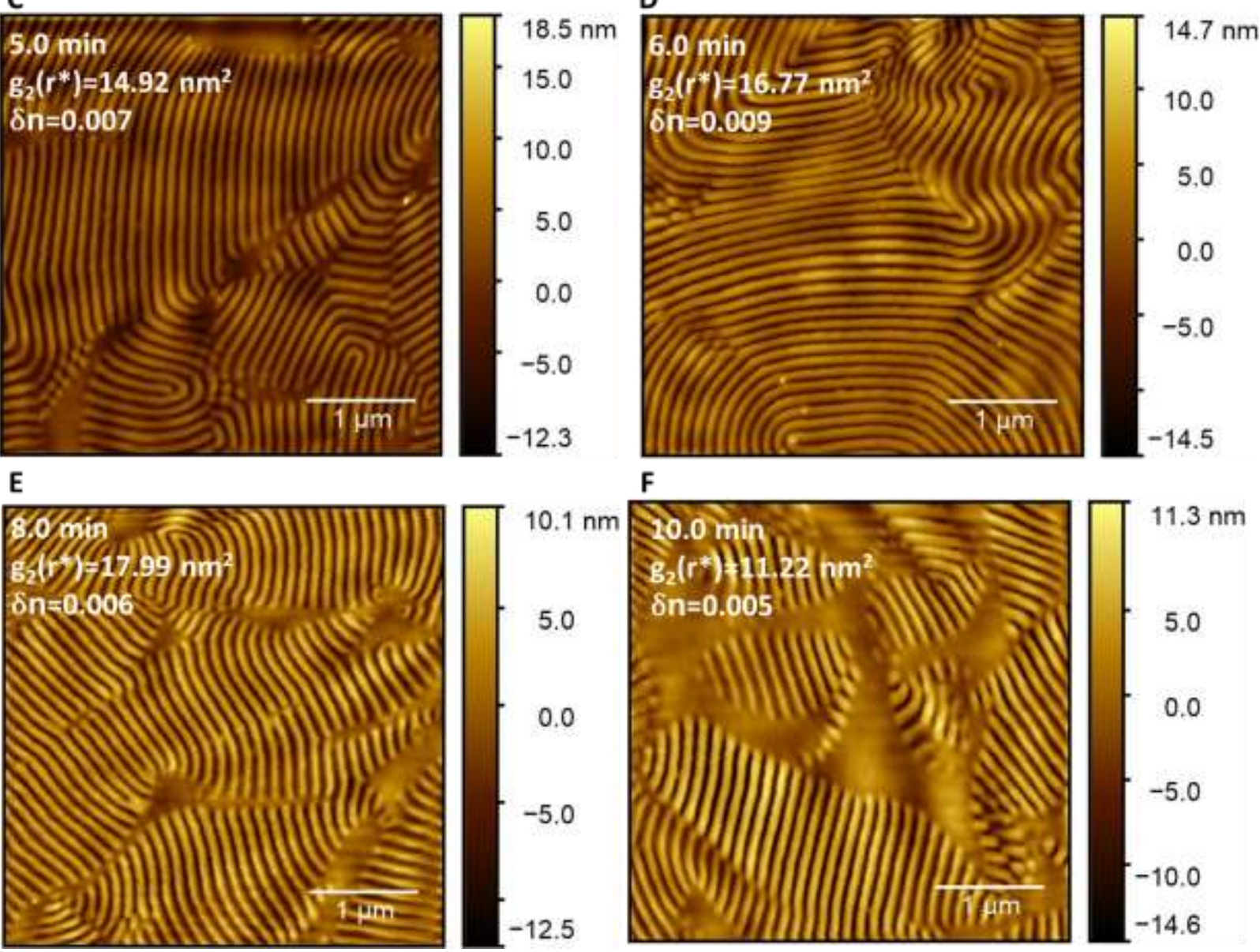

$-12.5$

$\mathbf{F}$

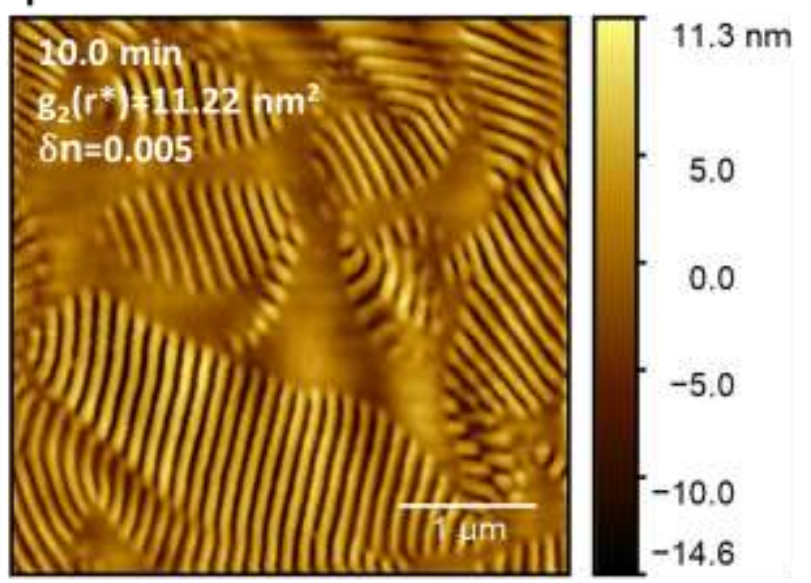

Figure S7: AFM images of 133k-b-132k PS-b-P2VP as a function of chloroform annealing time. The initial film thicknesses were all between $310 \mathrm{~nm}$ and $350 \mathrm{~nm}$. 


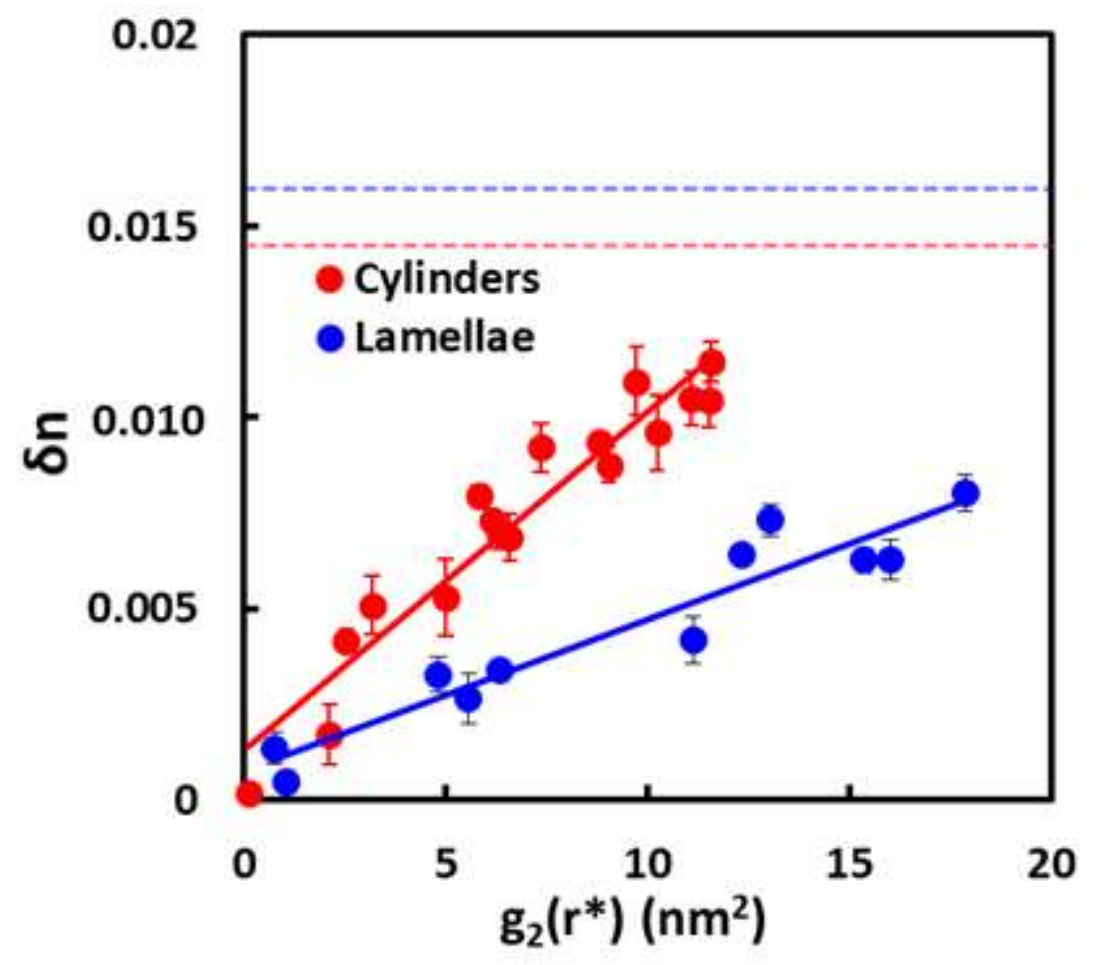

Figure S8: Correlation of the measured birefringence of both cylinder-and lamellae-forming BCPs with their surface ordering measured from AFM. The dashed colored lines are the maximum $\delta n$ for each geometry. 


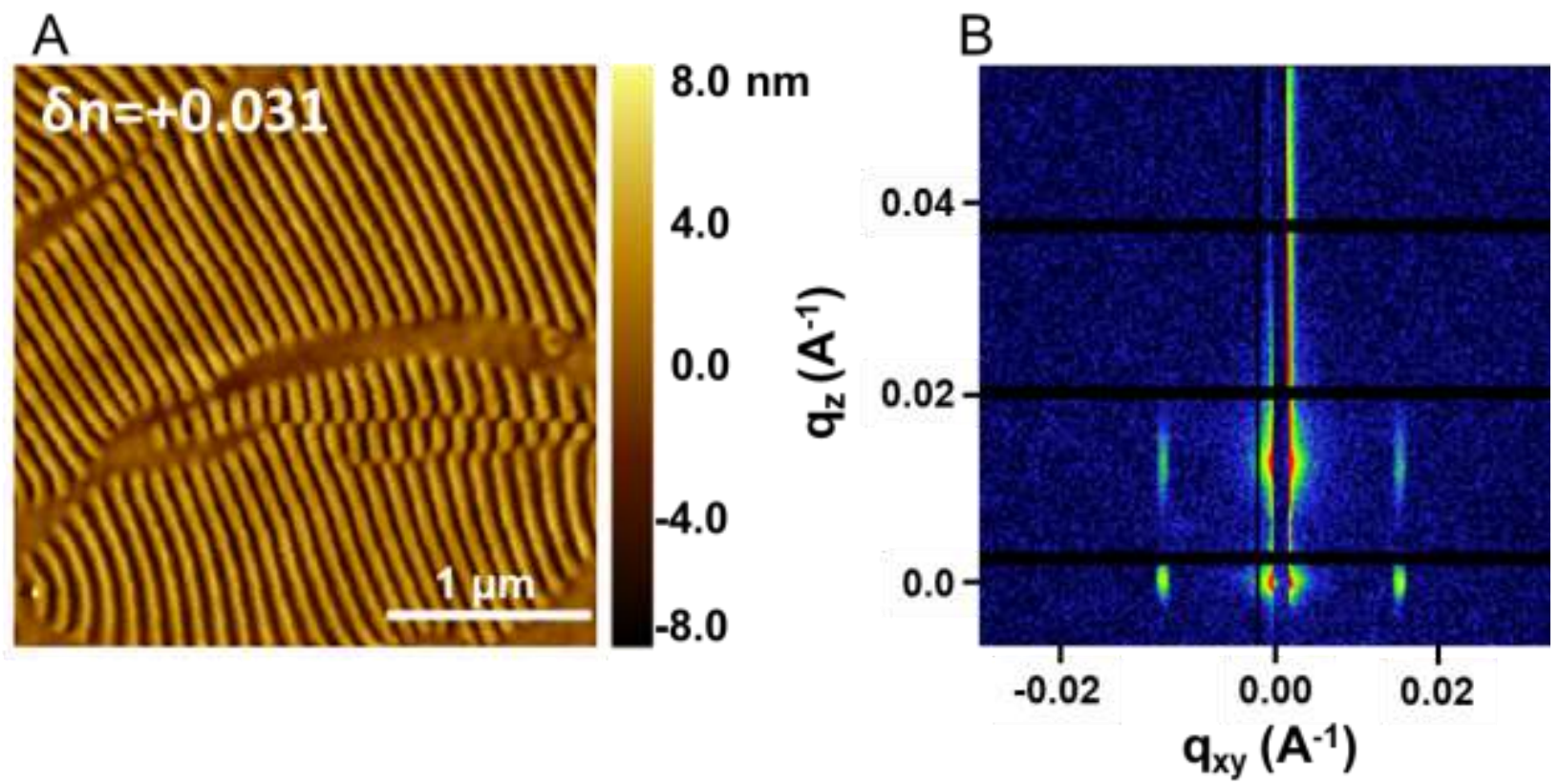

Figure S9: (A) AFM and (B) 2-D GISAXS data for PS-b-PMMA (38k-b-36k) (280nm) lamellae assembled perpendicular to the substrate thermal annealing for 48 hours at $190^{\circ} \mathrm{C}$. The scale bar in the image is $300 \mathrm{~nm}$. 

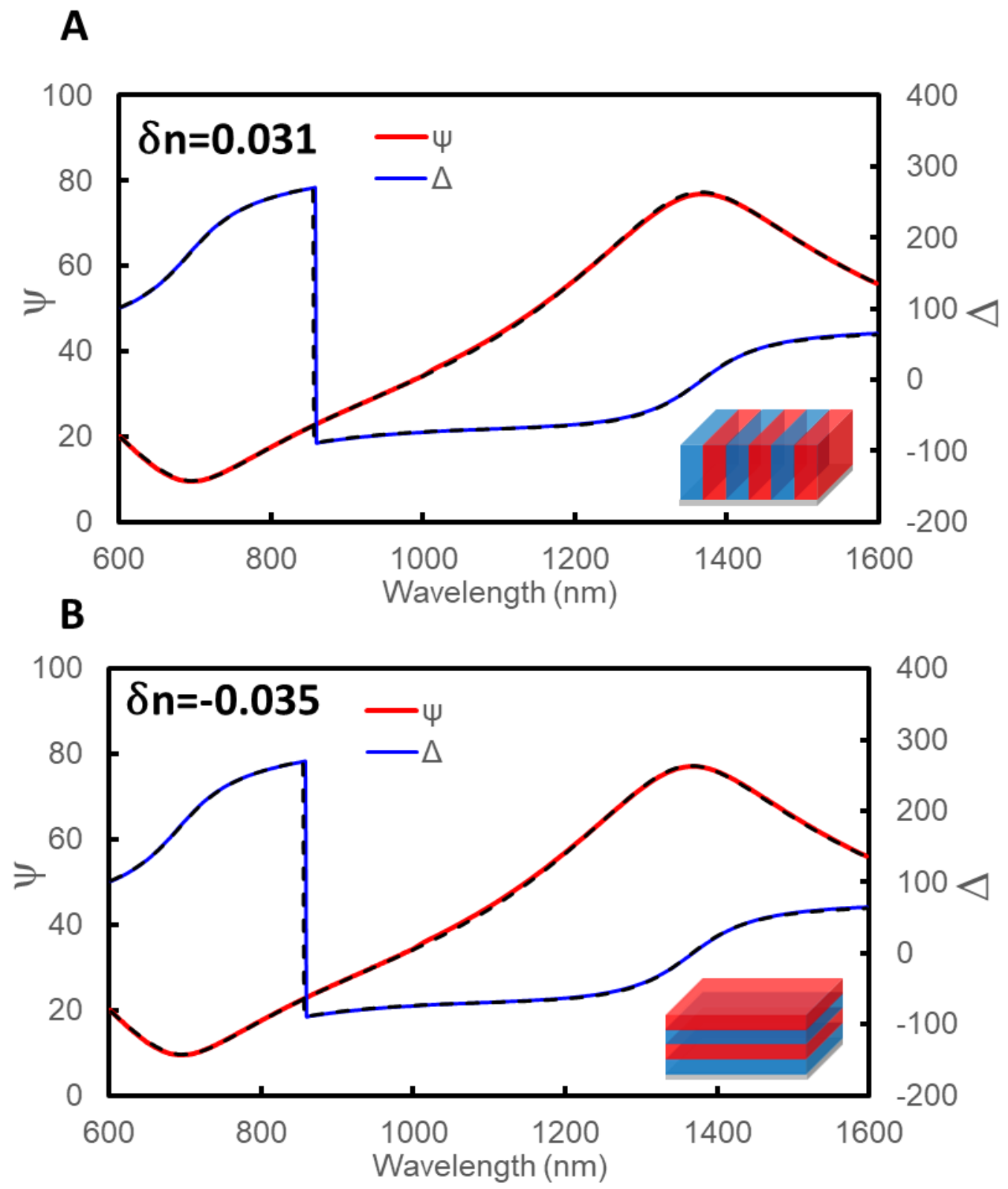

Figure S10: SE spectra of PS-b-PMMA (38k-b-36k) lamellae oriented (A) perpendicular to and (B) parallel to the substrate. The black dashed lines represent fits to a model with uniaxial birefringence. The MSEs of both fits are $<3$. The data in A corresponds to samples shown in Figure S9 and the data in B corresponds to the data in Figure 3 of the main text. 

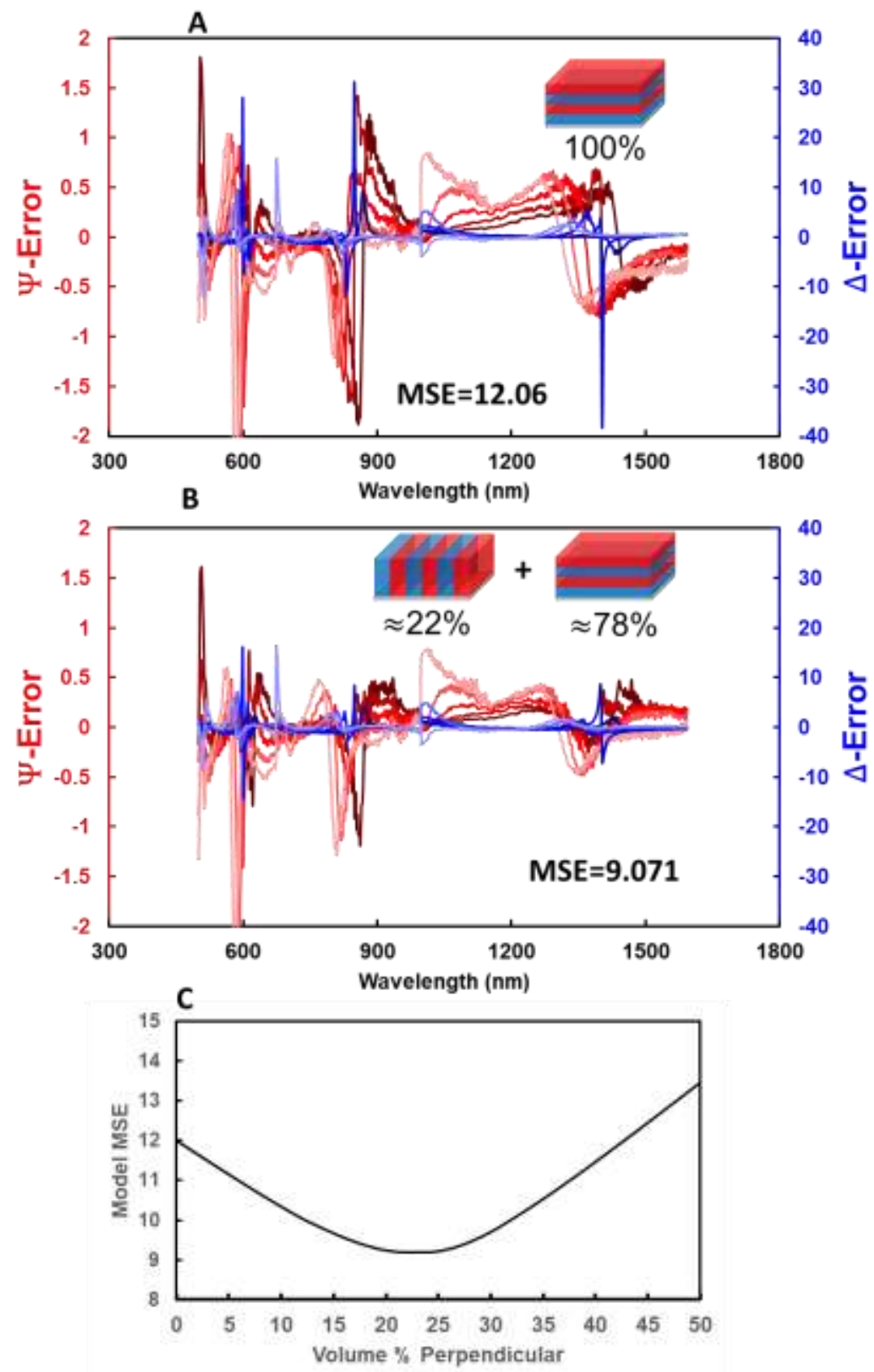

Figure S11: In-depth SE modeling of in-plane ordered lamellae. (A) Model MSE when the data is fit to inplane lamellae as the only structure present. (B) Model MSE when the data is fit to a volumetric average of in-plane and out-of-plane lamellae. The colors (from dark to light) denote $\Psi$ and $\Delta$ for increasing incident light angles from $55^{\circ}, 60^{\circ}, 65^{\circ}, 70^{\circ}$, and $75^{\circ}$. The MSE of the mixed-structure is approximately $25 \%$ lower than that of the single-structure. (C) MSE sensitivity to the volume fraction of minority perpendicular lamellae in the majority parallel lamellae. 

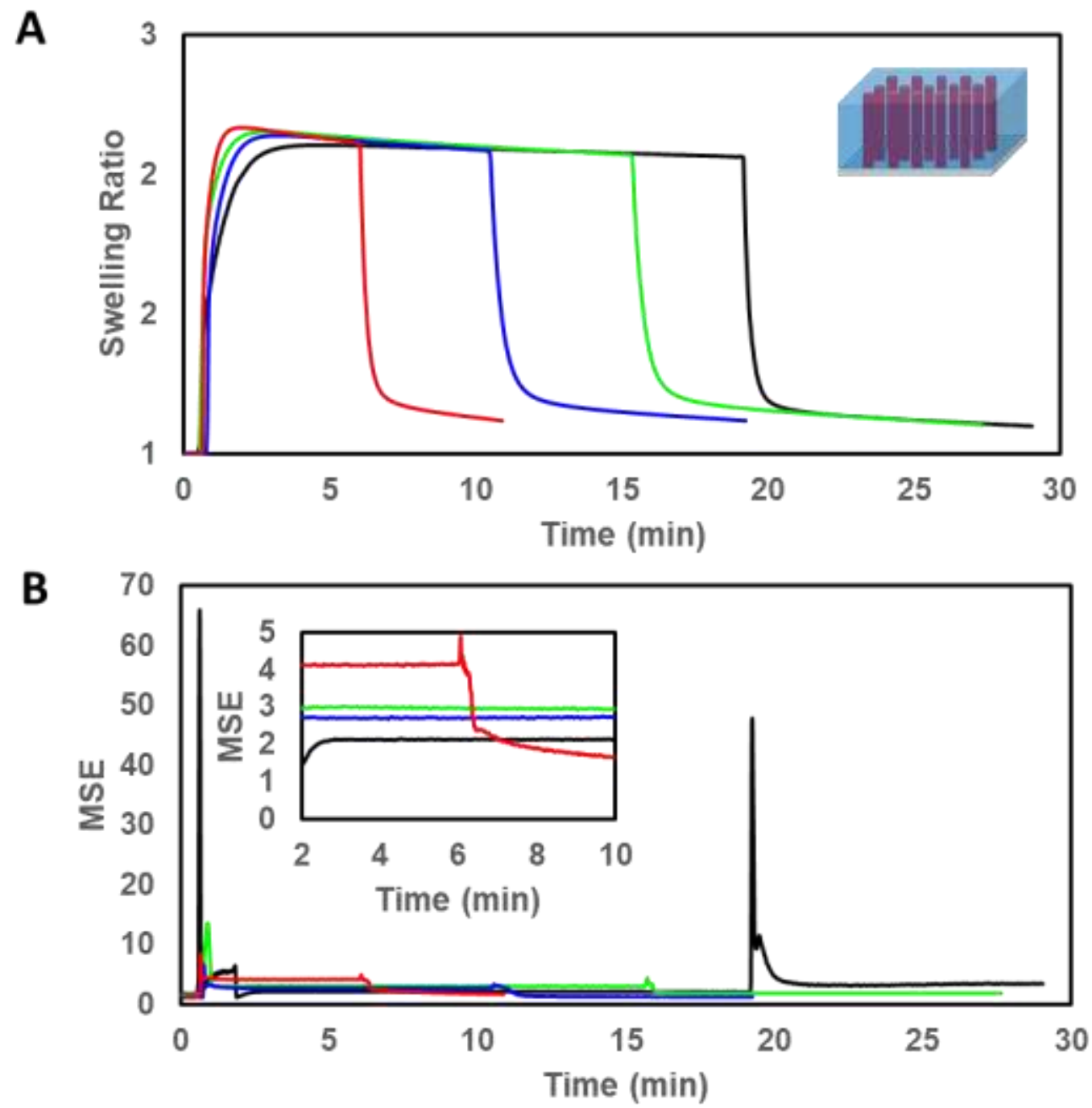

Figure S12: Swelling measurements of 48k-b-136k PS-b-P2VP films during in-situ solvent annealing with chloroform vapor. The colored lines correspond to Figure 4A in the main text; the colored lines represent films annealed for 5 (red), 10 (blue) and 15 (green) minutes. 
Ex-Situ
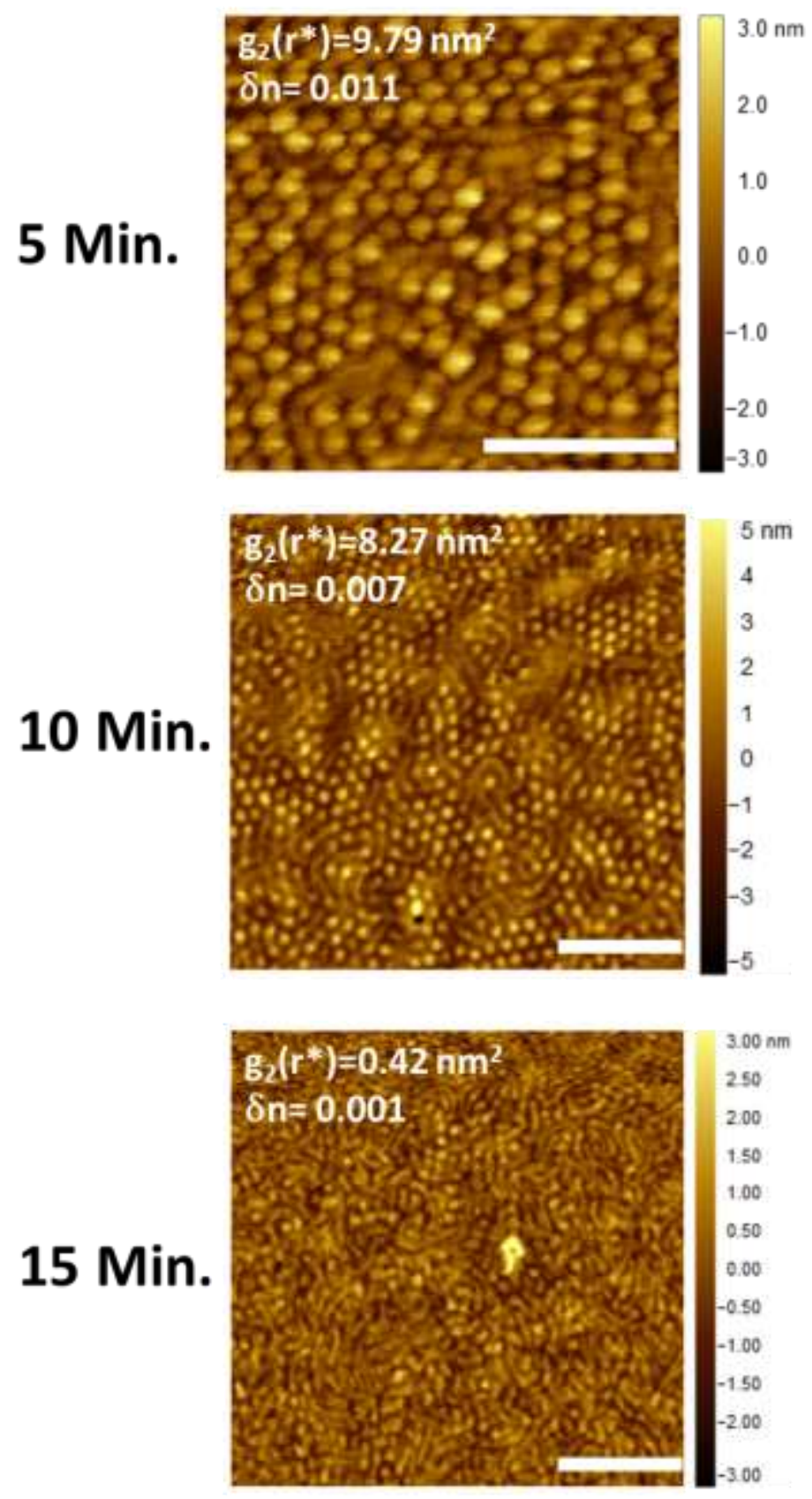

\section{In-Situ}
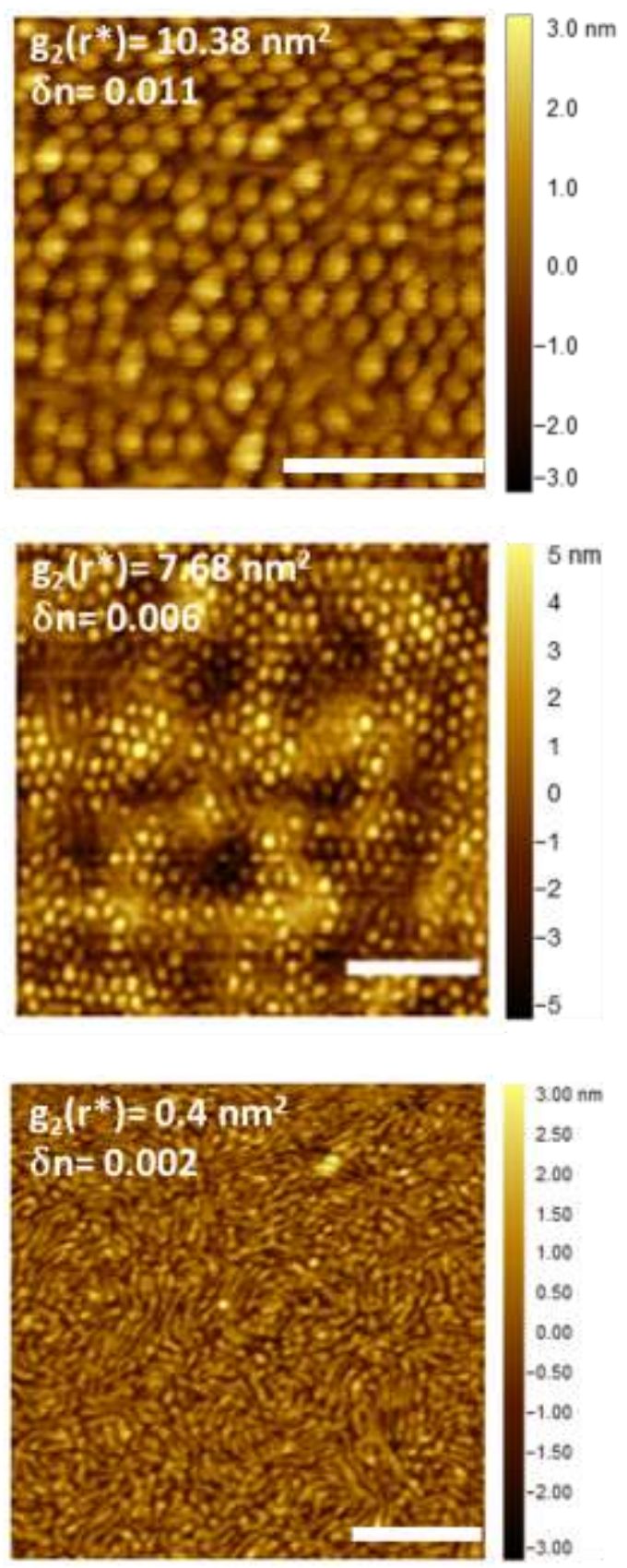

Figure S13: Morphological comparison of ex-situ and in-situ annealed BCP films (48k-b-136k) after 5, 10 , and 15 minutes of solvent annealing. The scale bars for the 5 minute images are $500 \mathrm{~nm}$, while the scale bars for the 10 and 15 minute images are $400 \mathrm{~nm}$. All initial film thicknesses are between $295 \mathrm{~nm}$ and $330 \mathrm{~nm}$. 
A

\section{As-Cast}

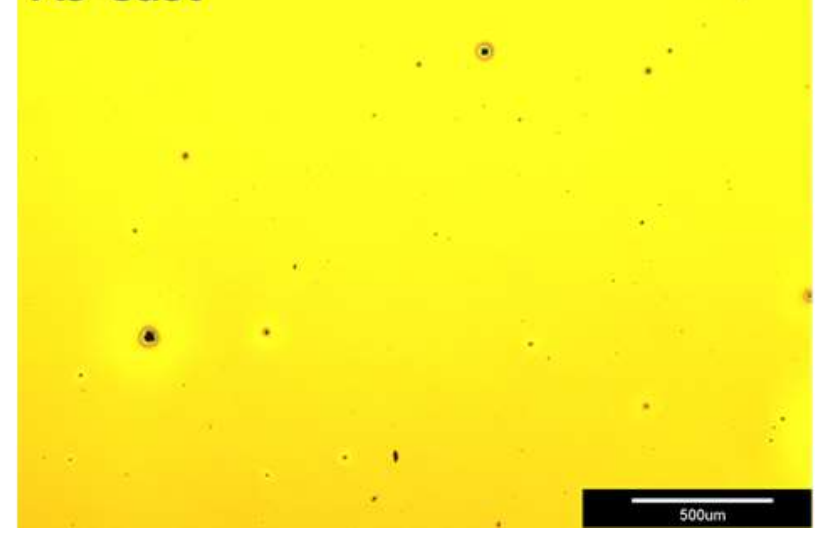

B

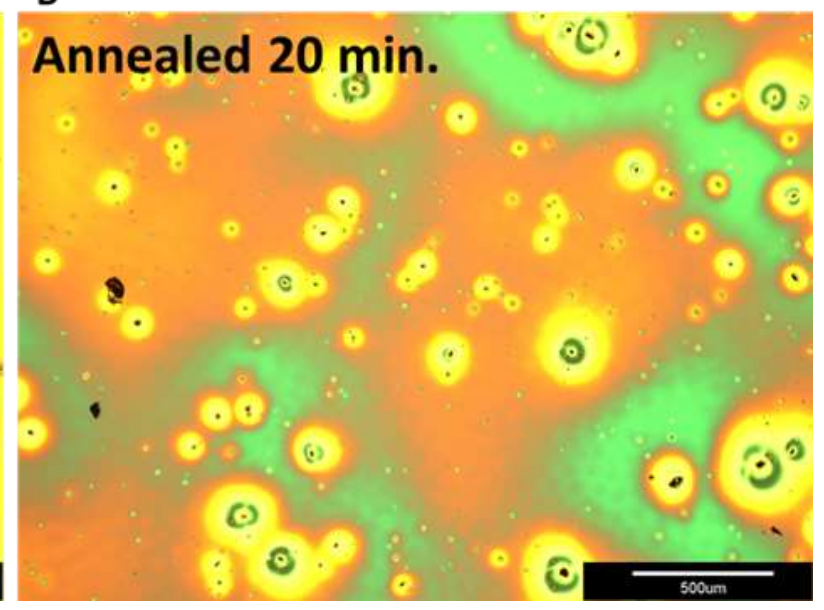

Figure S14: Optical microscopy images of a PS-b-P2VP film (48k-b-136k) (A) as-cast from solution and (B) after 20 minutes of chloroform vapor annealing. 


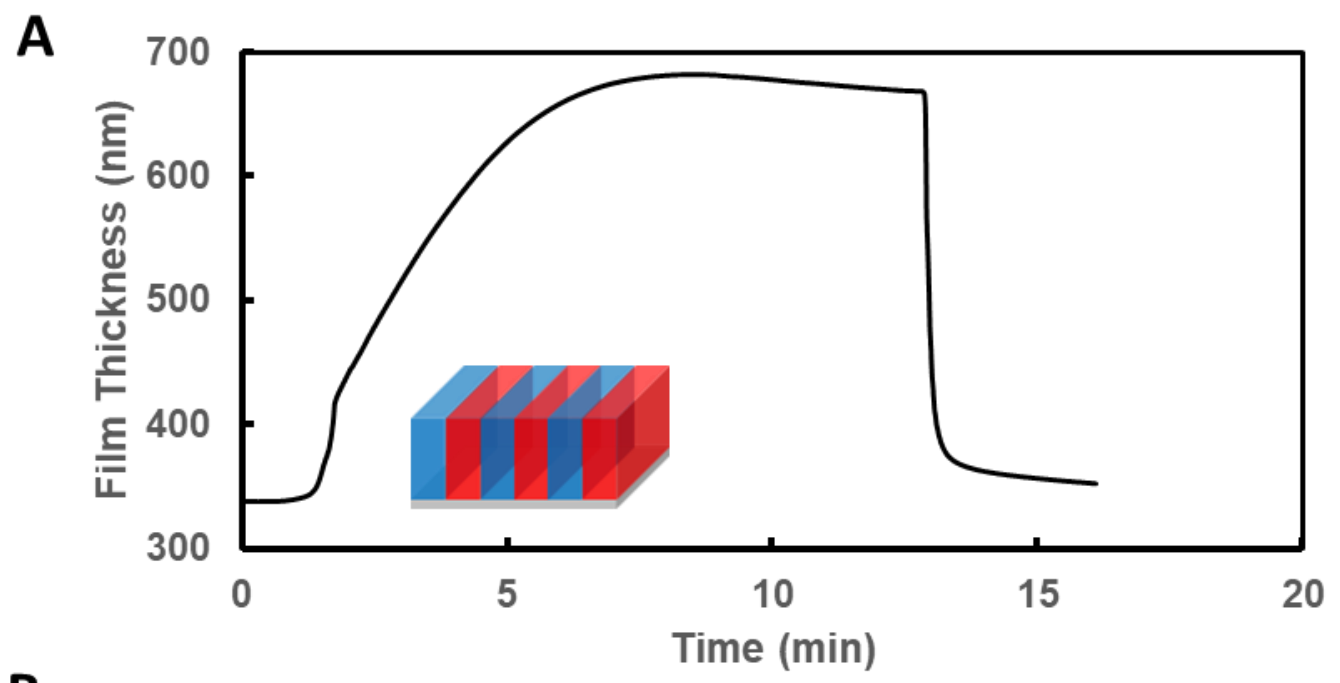

B

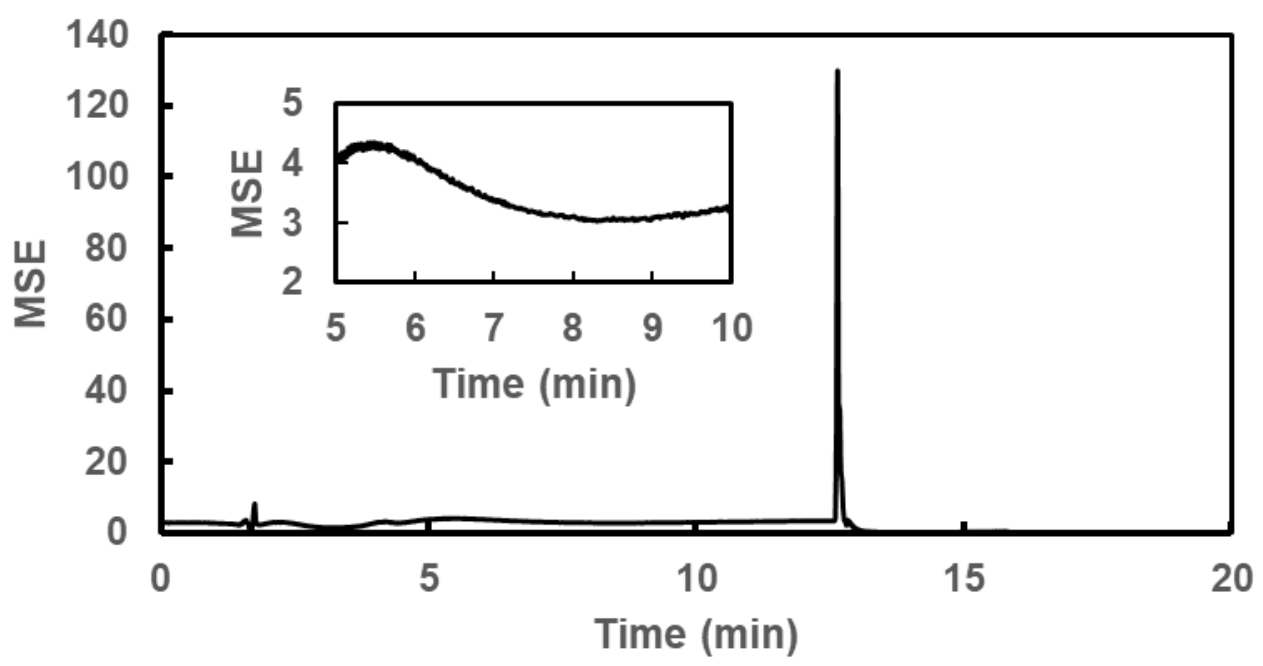

Figure S15: Swelling measurements of PS-b-P2VP (133k-b-132k) films during in-situ solvent annealing with chloroform vapor. 

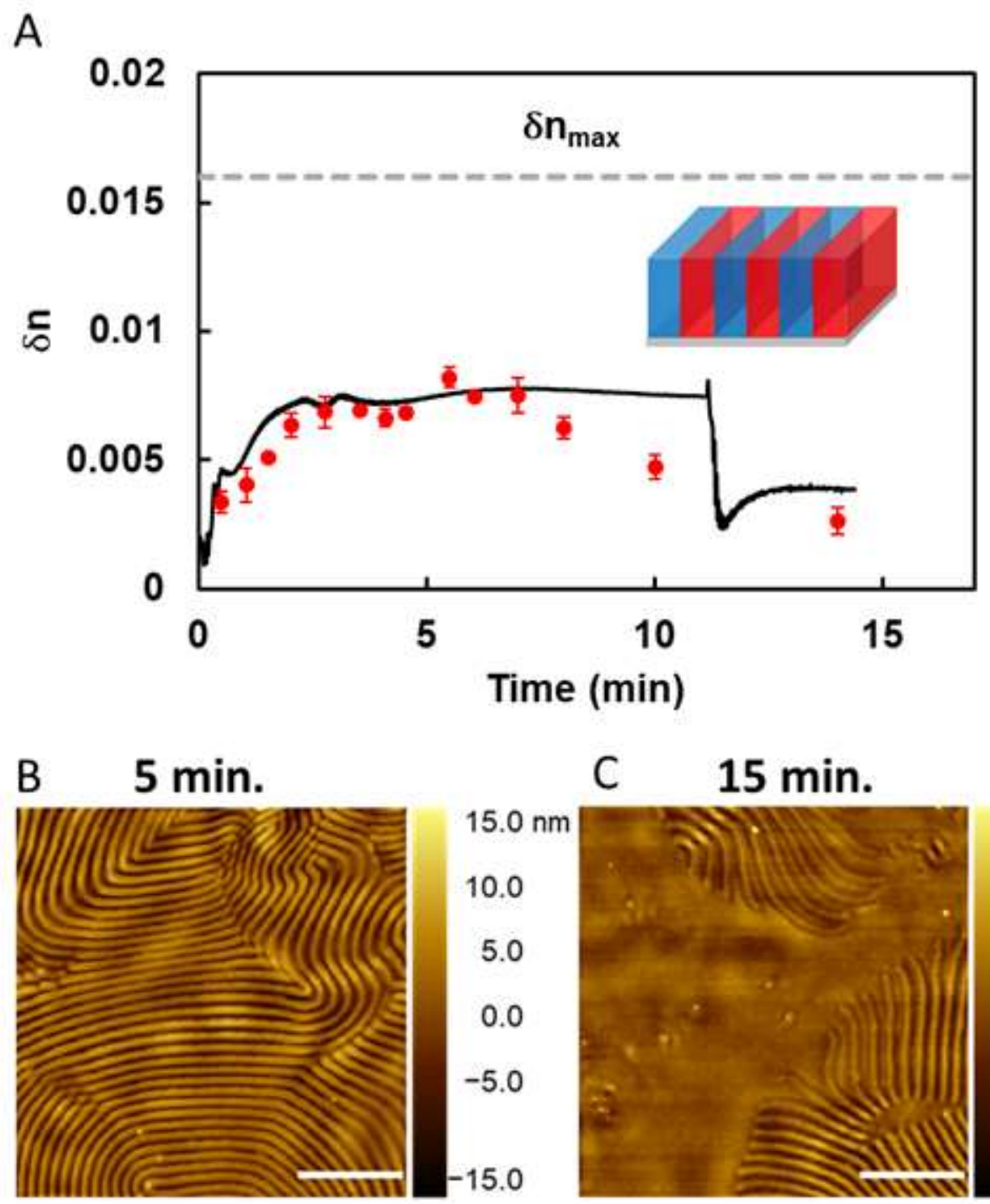

C $15 \mathrm{~min}$.

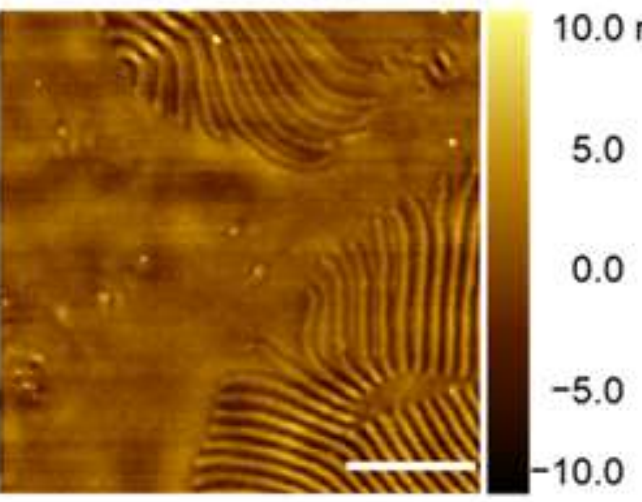

Figure S16: In-Situ SE of lamellae-forming BCPs. (A) measured birefringence as a function of chloroform annealing time in both ex-situ and in-situ geometries (380nm film thickness). (B,C) Ex-situ AFM images taken at different annealing times. Image scale bars are $1000 \mathrm{~nm}$. The initial film thickness was $305 \mathrm{~nm}$. 